\title{
TUMORAL MARKERS IN OVARIAN CANCER - A LITERATURE REVIEW
}

\author{
Nicolae Bacalbasa ${ }^{1}$, Alexandra Gireada ${ }^{2}$, Irina Balescu ${ }^{3}$ \\ 1"Carol Davila" University of Medicine and Pharmacy, Bucharest \\ 2"Carol Davila" Central Military Emergency Hospital, Bucharest \\ 3 "Ponderas" Hospital, Bucharest
}

\begin{abstract}
Ovarian cancer is one of the most lethal malignancies due to an aggressive biological behaviour of a tumor usually diagnosed in an advanced stage of the disease. Due to the fact that important differences in terms of survival have been observed between cases diagnosed in early stages when compared to those in FIGO stages III-IV, attention was focused on establishing which is the most appropriate laboratory diagnostic test which could help the clinician to diagnose and treat the disease in a less advanced stage. This is a literature review of the most frequently used tumor markers and scores used in ovarian cancer diagnostic and follow up.
\end{abstract}

Keywords: ovarian cancer, CA 125, HE 4, ROMA score, OVA1

\section{INTRODUCTION}

Ovarian cancer represents one of the most frequently seen malignancies in women and the most important genital cancer related death cause in women worldwide. Ovarian epithelial cancer represents the most frequent hystoppathological subtype, accounting for almost $85 \%$ of cases of ovarian cancer. Due to the high incidence of this malignancy and due to its' very aggressive biological behaviour, multiple studies focused on early diagnose, creation of screening tests and establishing an appropriate follow-up protocol for these patient. Although the most appropriate screening test remains to be established at this moment multiple diagnostic scores have been imagined (1).

\section{TUMOR MARKERS USED IN PATIENTS WITH OVARIAN CANCER}

\section{CA 125}

The most common used marker for ovarian cancer is CA 125. A strong correlation can be estab- lished between the sensibility of this marker and FIGO stage of the disease. Cases diagnosed in FIGO stage I report high values of CA 125 in up to $50 \%$ cases while in more advanced stages of the disease (FIGO stage III-IV) proportion of positive results reaches almost $90 \%$. However, the most important deficiency of CA 125 is a low diagnostic specificity, high values being also seen in other gynecologic malignancies such as endometrial cancer, endocervical cancer, in other epithelial tumors such as pulmonary adenocarcinoma or non-epithelial cancer - lymphomas $(1,2)$.

\section{CA 125, HE4, ROMA score, RMI}

A more recent tumor marker used in ovarian cancer is HE4, with a comparable sensibility with CA 125 but with a higher specificity

A study conducted by Escudero et al in 2011 realised a comparison between HE4 and CA 125 in order to diagnose and differentiate between malignant and benign tumors. The study included 101 healthy subjects, 535 patients with benign disorders and 423 patients with malignant tumors. 
Cut-off values for CA 125 and HE4 were $35 \mathrm{kU} / \mathrm{L}$ and $140 \mathrm{pmol} / \mathrm{L}$ respectively. Abnormal values of HE4 and CA 125 were seen in $1,1 \%$ respectively $9,9 \%$ in healthy subjects and $12.3 \%$ respectively $37 \%$ in cases with benign disorders. In cases with benign ovarian conditions HE4 was positive in $1.3 \%$ of cases while CA 125 was positive in 33,2 . High values of HE4 in patients with malignant diseases were most frequently seen in gynecologic and pulmonary cancer, in contrast with CA 125 which was found in other various epithelial and non-epithelial tumors.

The concentration of the both tumoral markers was also in strong correlation with FIGO stage of ovarian cancer, with significantly higher values in advanced stages (FIGO stage III-IV) (CA $125-\mathrm{p}=$ $0,001, \mathrm{HE} 4-\mathrm{p}=0,004)$. Also, a strong correlation was established between the histopathological subtype and tumoral markers values, with a significantly higher concentration in serous epithelial ovarian adenocarcinoma (CA $125-\mathrm{p}=0,003$, HE4 $-\mathrm{p}=0.009)(2)$.

A study conducted by Wen-Ting Chen et al compared the role of the two tumoral markers in prediction and follow-up of patients with ovarian cancer. A number of 123 cases with ovarian cancer and 174 control cases were selected; in all cases HE4 was measured and ROMA score was calculated; based on these values, two subgroups were created: a high and a low malignancy risk subgroup. Postoperative lower values of HE4 were stronger correlated with an improved outcome while persistent high values of HE4 were associated with a higher rate or recurrence and a poor overall survival. A positive postoperative response was defined as a $50 \%$ decrease of tumor markers' value at two successive dosages. ROMA scores were calculated and the sensibility was $88.9 \%$ for the first subgroup and $91.3 \%$ for the second subgroup. Cut-off values were established for a $75 \%$ specificity (3).

A study conducted in 2009 by Montagana et al determined HE4 and CA 125 values for patients with benign and malignant pelvic tumors. In order to compare the efficacy of the 2 markers, the study departure point was the low sensibility of CA 125 in early ovarian cancer and HE4's capacity of supraexpression in ovarian cancer. The study included 99 cases with gynecological malignancies (46 cases with ovarian cancer, 39 cases with endometrial cancer and 14 cases with cervical cancer) and 40 cases with benign disorders ( 22 cases with endometriosis and 18 cases with benign ovarian tumors); a control group of 12 cases was also involved. The mean seric concentrations for CA 125 and HE 4 were significantly higher for cases with ovarian malignancies when compared to benign disorders and healthy subjects, with a significantly higher difference for HE4 (with a reported sensibility of $98 \%$ and $100 \%$ specificity) (4).

In order to increase the rate of complete cytoreduction in cases with advanced ovarian cancer, prediction scores involving clinical data (menopausal status), imagistic studies (ultrasonography) and tumor markers (CA 125, HE4) were imagined. The most common scores are RMI (risk of malignancy index) - based on ultrasonographic data, menopausal status and CA 125 values, ROMA score involving menopausal status, CA 125 and HE 4, OVA 1 score - involving CA 125, $\beta 2$ microglobulin, A1 apo-lipoprotein, transtirenine and transferine and LR 2 - an ultrasonographic model of prediction (5).

Advantages of using an algorithm based on seric values of tumoral markers consist in eliminating any subjective factors and creating an objective protocol which can be safely applied worldwide $(6,7)$.

RMI was created by Jacobs et al in order to differentiate cases with high malignancy rates; however the main disadvantage of the score is the low specificity of CA 125 especially in premenopausal women. Due to this aspect RMI is used most in postmenopausal women (8).

Association of HE4 dosage and ROMA score model provides a better screening value. A study published by Moore et al in 2007 was conducted on 259 patients with pelvic tumors who were candidates for surgery. Preoperative tests were performed in 233 cases (67 patients with ovarian cancer and 166 cases with benign ovarian conditions) and involved CA125, SMRP, HE4, CA72-4, activine, inhibine, osteopontine, EGF (epidermal growth factor) and Her2. HE4 was associated with the highest sensibility $(72.9 \%)$ and specificity (95\%). HE4 was also the best marker in early stage ovarian cancer while its' association with any other marker was not associated with a higher sensibility (9).

The same authors also demonstrated that determination of seric concentrations for both HE4 and CA 125 can differentiate endometriosis from malignant conditions with a reported sensibility of $79 \%$ (compared to $64 \%$ sensibility for CA 125 alone and $71 \%$ sensibility for HE 4 alone) (10).

Another study which demonstrated the utility of CA 125 associated with HE 4 in order to differentiate ovarian cancer from ovarian endometriosis was the one conducted by Huhtein et al. They reported that the association of CA 125 and HE 4 dosages 
had an accuracy of $96.3 \%$ and sensibility of $92.9 \%$ in order to differentiate ovarian cancer from healthy subjects, while the same measurements had an accuracy of $94 \%$ and sensibility of $78.6 \%$ in order to differentiate ovarian cancer from ovarian endometriosis (11).

In another study conducted by Moore et al ROMA score was calculated preoperatively and according to the obtained values, patients were classified on two groups: high and low malignancy risk; sensibility, specificity, positive and negative predictive values were estimated. In post-menopausal women the sensibility rate was $92.3 \%$ and specificity was $76 \%$, while in pre-menopausal subjects sensibility was $100 \%$ and specificity was $74,2 \%$. When determined on the entire lot, ROMA score reported a sensibility of $93.8 \%$, a specificity of $74.9 \%$ and a negative predictive value of $99 \%$. For a pre-established value of $75 \%$ specificity, ROMA score indicated a sensibility of $94 \%$ in differentiation between malignant and benign lesions and a sensibility of $85 \%$ for identifying early stages of ovarian cancer. The accuracy of ROMA score was higher when compared to RMI score (6).

A study conducted by Bandiera et al involved 419 cases: 140 healthy subjects, 131 patients with benign ovarian cysts, 34 with endometriosis lesions and 114 cases with epithelial ovarian cancer and tried to establish the role of HE4 and ROMA score in diagnostic and follow up of patients with ovarian malignancies. The sensibility and specificity in differentiating benign from malignant conditions in premenopausal women were $92,3 \%$ and $59,4 \%$ for CA $125,84.6 \%$ and $94.2 \%$ for HE4 and $84.65 \%$ respectively $81.2 \%$ for ROMA score; in postmenopausal women values were $94.3 \%$ and $82.3 \%$ for CA $125,78.2 \%$ and $99 \%$ for HE4, respectively $93.1 \%$ and $84.4 \%$ for ROMA score. In patients with epithelial cancer HE4 and ROMA scores were useful in order to differentiate poor differentiated tumors from well differentiated subtypes, with $\mathrm{p}$ values $<0,001$ for both markers, while CA 125 did not report significant modifications between the two histopathological subtypes $(p=0.0579)$. In multivariate analysis, HE4 and ROMA score were independent prognostic factors for overall survival and disease free survival (7).

Sandri et al conducted a study on 349 pre- and post-menopausal patients with pelvic tumoral masses and reported significantly higher values for CA 125 and HE 4 in cases with ovarian cancer $(p<0.0001)$; when evaluating the accuracy, CA 125 reported a score of $89.9 \%$ in pre-menopausal women, while ROMA score reported a value of $93.3 \%$ for post-menopausal subjects (12).

\section{OVA1}

In September 2009 FDA approved OVA 1 testing in patients with pelvic masses which were proposed for surgery. In fact, OVA 1 determines serum concentrations for proteins which report abnormal values in patients with ovarian cancer: CA 125 II, transtirenine (pre-albumin), A1 Apo lipoprotein, $\beta 2$-microglobuline and transferine and generates a numeric score between $0-10$ which indicates the possibility of malignancy of a pelvic mass. Premenopausal women with a reported score $>5$ and post-menopausal women with a reported score $>4$ are considered to be high risk patients. These cutoff values were established after examination of 516 patients, with a mean age of 52 years, $46 \%$ of them post-menopausal, with a pelvic mass diagnosed by imagistic studies and who were proposed for surgery within 3 months. OVA 1 test reported a sensibility of $93 \%$, a specificity of $43 \%$, a positive predictive value of $42 \%$ and a negative predictive value of malignancy of $93 \%$. In pre-menopausal women replacing CA 125 with OVA 1 increased the sensibility rate from $60 \%$ to $89 \%$ and modified the negative predicting value from $90 \%$ to $94 \%$. In postmenopausal women replacing CA 125 with OVA 1 increased the sensibility rate from $81 \%$ to $98 \%$ and the negative predictive value from $85 \%$ at $96 \%$. In epithelial ovarian tumors OVA 1 sensibility was $99 \%$, while in non-epithelial cancer sensibility was $78 \%$; a sensibility of $75 \%$ was encountered for low-malignant tumors while ovarian metastases had a sensibility of $94 \%$ (13).

\section{CONCLUSIONS}

Although a perfect marker for positive diagnostic of ovarian cancer has not yet been found, it seems that association of HE4 and creation of ROMA score significantly improved the positive preoperative diagnostic of this aggressive gynecologic malignancy. Recent studies involving OVA 1 seem to provide encouraging results in order to improve the pre-operative positive diagnostic of ovarian cancer. 


\section{REFERENCES}

1. The National Horizon Scanning Centre, University of Birmingham, United Kingdom UPDATE: OVA $1^{\mathrm{TM}}$ test for the assessment of suspected ovarian cancer, February 2011

2. Escudero J.M., Auge J.M., Filella X. et al. Comparison of serum human epididymis protein 4 with cancer antigen 125 as a tumor marker in patients with malignant and nonmalignant diseases. Clin Chem . 2011; 57:1534-1544.

3. Chen W.T., Gao X., Han X.D. et al. HE4 as a serum biomarker for ROMA prediction and prognosis of epithelial ovarian cancer. Asian Pac J Cancer Prev. 2014; 15:101-105.

4. Montagnana M., Lippi G., Ruzzenente O. et al. The utility of serum human epididymis protein 4 (HE4) in patients with a pelvic mass. J Clin Lab Anal. 2009; 23:331-335.

5. Wilailak S., Chan K.K., Chen C.A. et al. Distinguishing Benign from Malignant Pelvic Mass Utilizing an Algorithm with HE4, Menopausal Status, and Ultrasound Findings. J Gynecol Oncol. 2014.

6. Moore R.G., McMeekin D.S., Brown A.K. et al. A novel multiple marker bioassay utilizing HE4 and CA125 for the prediction of ovarian cancer in patients with a pelvic mass. Gynecol Oncol. 2009; 112:40-46.

7. Bandiera E., Romani C., Specchia C. et al. Serum human epididymis protein 4 and risk for ovarian malignancy algorithm as new diagnostic and prognostic tools for epithelial ovarian cancer management. Cancer Epidemiol Biomarkers Prev. 2011; 20:2496-2506.

8. Hellstrom I., Raycraft J., Hayden-Ledbetter M. et al. The HE4 (WFDC2) protein is a biomarker for ovarian carcinoma. Cancer Res. 2003; 63:3695-3700.

9. Moore R.G., Brown A.K., Miller M.C. et al. The use of multiple novel tumor biomarkers for the detection of ovarian carcinoma in patients with a pelvic mass. Gynecol Oncol. 2008; 108:402-408.

10. Moore R.G., Miller M.C., DiSilvestro P. et al. Evaluation of the diagnostic accuracy of the risk of ovarian malignancy algorithm in women with a pelvic mass. Obstet Gynecol. 2011; 118:280-288.

11. Huhtinen K., Suvitie P., Hiissa J. et al. Serum HE4 concentration differentiates malignant ovarian tumours from ovarian endometriotic cysts. Br J Cancer. 2009; 100:1315-1319.

12. Sandri M.T., Bottari F., Franchi D. et al. Comparison of HE4, CA125 and ROMA algorithm in women with a pelvic mass: correlation with pathological outcome. Gynecol Oncol. 2013; 128:233-238.

13. Abraham J. Section Editor, OVA1 test for preoperative assessment of ovarian cancer, Commun Oncol. 2010; 7:249-251. 